\title{
Estudio comparativo entre el método calorimétrico y el de estimación matemática para la determinación energía bruta en subproductos agrícolas
}

\author{
Comparative study between the calorimetric method and the mathematical \\ estimation method for the determination of gross energy in agricultural by \\ products
}

Angélica Chugñay Cargua. ${ }^{1}$, Marcelo Ramos Flores. ${ }^{2}$, Manfredy Chugñay Cargua. ${ }^{3} \&$ María Eugenia Ramos Flores. ${ }^{4}$

Recibido: 22-06-2019 / Revisado: 28-07-209 /Aceptado: 09-08-2019/ Publicado: 10-09-2019

\begin{abstract}
DOI: https://doi.org/10.33262/cienciadigital.v3i3.4.831

Two methods used to determine the gross energy value of food: calorimetric and mathematical estimation were compared, in order to determine if there is statistical similarity between their results, and thus consider them both equivalent. To this end, duplicate samples of eight agricultural by-products destined for animal feed were prepared, one of which was subject to proximal analysis for later application of the mathematical estimation method, and the other was analyzed directly in bomb-calorimetric. Both sets of results were characterized by descriptive statistics, showing both normal distribution with non-homogeneous variances; so, they were compared using the $t$ test with the Welch variation. The test determined that the means of the results of both methods show a significant difference; in addition, the existence of positive bias was observed, where the calorimetric method delivers numerically higher results than those of the mathematical estimation method.
\end{abstract}

Keywords: Gross energy, comparison of methods, proximal analysis, calorimetric bomb, statistical bias

\section{Resumen}

Se compararon dos métodos utilizados para determinar el valor de energía bruta de alimentos: calorimétrico, y de estimación matemática, para determinar si existe similaridad estadística entre sus resultados, y así considerarlos equiparables. Para ello se prepararon muestras duplicadas de ocho subproductos agrícolas destinados a alimentación animal, una de las cuales se sometió a análisis proximal para posterior aplicación del método de estimación matemática, y la otra se analizó directamente en una bomba calorimétrica. Ambos conjuntos de resultados se caracterizaron mediante estadísticos descriptivos, mostrando ambos distribución normal con varianzas no-homogéneas; por lo que se compararon mediante test $\mathrm{t}$ con la variación de Welch. El test determinó que las medias de los resultados de ambos

\footnotetext{
${ }^{1}$ Escuela Superior Politécnica de Chimborazo, angelica.chugnay@espoch.edu.ec

2 Escuela Superior Politécnica de Chimborazo,jmramos@espoch.edu.ec

${ }^{3}$ Escuela Superior Politécnica de Chimborazo, machugnay@espoch.edu.ec

4 Universidad Estatal Amazónica,mramos@uea.edu.ec
} 
métodos presentan diferencia significativa, observándose además la existencia de sesgo positivo, donde el método calorimétrico entrega resultados numéricamente mayores a los del método de la estimación matemática.

Palabras clave: energía bruta, comparación de métodos, análisis proximal, bomba calorimétrica, sesgo estadístico.

\section{Introducción}

El Ecuador en sus tres regiones es un país altamente agrícola que posee recursos vegetales disponibles para su utilización en la alimentación de animales; residuos que no son utilizados por desconocimiento del valor nutricional y energético. En los últimos años se ha puesto atención al uso de residuales agrícolas para la alimentación de ganado porque, además de maximizar la eficiencia del uso de los recursos, disminuye costos de producción, mismos que representan entre un 60 a 70\% del costo total de producción (Torres Navarrete, y otros, 2017), reduce la competición por alimento entre el hombre y el ganado. (García, Henry, \& Schulmeister, 2015) y lleva a una reducción del impacto ambiental de la actividad agroganadera (Núñez Torres, 2017).

La información sobre el valor energético de alimentos es esencial para ejecutar estudios nutricionales sobre dietas destinadas a tratar la malnutrición calórica o la obesidad en animales (Hernández \& Sastre, 1999). Los alimentos pueden valorarse inicialmente por sus propiedades organolépticas, para evaluar si están en condiciones de ser utilizados, así también, por su composición química, para conocer si responden con las especificaciones establecidas y su aporte de energía. Las principales fuentes de energía en los alimentos son las proteínas y el extracto etéreo, la energía bruta es la máxima energía potencial presente en un alimento (Hernández \& Sastre, 1999).

La "energía bruta", EB, es la energía química total almacenada en la materia orgánica que constituye los alimentos. Se expresa como calor de combustión por unidad de peso del alimento (Caravaca Rodríguez, Castel Genís, \& Otros, 2005). De acuerdo a la primera ley de termodinámica indica que todas las diferentes formas energía pueden transformarse en calor, de allí que, cuando una sustancia se combustiona, el calor que se desprende es equivalente a la energía bruta almacenado en dicho alimento (Cañas Cruchaga, Aguilar González, García Gómez, Quiroz, \& otros, 1992).

La valoración del contenido de energía bruta presente en los alimentos puede ser determinada de forma directa utilizando una bomba calorimétrica, donde el alimento se combustiona (Santos, 2010); o por el método de estimación matemática, partiendo de resultados de un análisis químico proximal previo (Mora Brautigan, 2007). Entre estas dos opciones, el método calorimétrico toma 
menos tiempo, es más económico y no demanda de análisis previos, pero también es considerado como un método "grueso" o "rápido" (Mora Brautigan, 2007)

No obstante, antes de considerar que el método calorimétrico pueda reemplazar a la estimación matemática, usada tradicionalmente y que da resultados ampliamente aceptados; se hace necesario comparar estadísticamente sus resultados.

\section{Importancia}

Se carece de investigaciones que analicen a nivel estadístico si los dos métodos presentan similaridad o diferencia entre ellos, además, a la fecha de realización del estudio, en Ecuador no se disponen de estudios en donde se haya determinado la cantidad de energía bruta de subproductos no convencionales con interés para la alimentación animal.

Estudios similares realizados para comparar el método de estimación matemática y el calorimétrico para la determinación del contenido energético de alimentos, como el de (Batres Gracias, 2004) determinó que los valores de energía bruta obtenidos por estimación matemática, partiendo del análisis químico proximal fueron menores a los valores cuantificados por el calorimétrico, en el caso de productos para alimentación de cerdos. Por otra parte, (Posada, Rosero, Rodríguez, \& Costa, 2012), en el caso de alimentos para rumiantes, determinaron por medio de la prueba de Tukey que los dos métodos diferían entre sí, con valores calorimétricos mayores que los procedentes de la estimación matemática.

La investigación realizada consistió en análisis de similaridad estadística entre las medias de valores de energía bruta de ocho subproductos agrícolas no-convencionales, de los cuales se tomaron muestras por duplicado, una de las cuales se analizaron por calorimetría y la otra por estimación matemática. Los resultados permitieron responder a la interrogante si, ambos métodos puedan considerarse equiparables desde un punto de vista de los resultados obtenidos al medir energía bruta.

\section{Metodología}

La población de estudio estuvo constituida por ocho subproductos agrícolas no-convencionales de la sierra ecuatoriana: trabajando un total de 44 muestras, compuestas por: tronco de brócoli: 12, zanahoria: 13 , chaqui sara: 2 , hoja de chocho: 6 , hoja de maíz: 3 , hoja de plátano: 4, polvillo de quinua: 2, hoja de camote: 2. Las muestras fueron recolectadas y seleccionadas según su biodisponibilidad, y sometidas a tratamiento previo de secado y molienda, separando una porción para aplicación del método calorimétrico y otra para estimación matemática. 
La investigación se realizó en la Escuela Superior Politécnica de Chimborazo, en la ciudad de Riobamba, Ecuador. El tratamiento de muestras y las mediciones se efectuaron en el laboratorio de Bromatología de la Facultad de Ciencias Pecuarias y el procesamiento y análisis de los datos en la Escuela de Ingeniería Química de la Facultad de Ciencias.

\section{Análisis químico proximal}

El análisis químico proximal, es probablemente el método más utilizado para expresar la calidad nutritiva de un alimento, midiendo seis parámetros: humedad, cenizas, extracto etéreo (EE), fibra bruta (FB), proteína bruta (PB), y extracto libre de nitrógeno (ELN) (Barrera, Tapia, \& Monteros, 2004). La medición se realizó sobre muestras secas de los mencionados subproductos.

\section{Energía bruta por estimación matemática}

Para determinar el valor de energía bruta por el método de estimación matemática se partió de los resultados del análisis químico proximal. Para ello se operaron los valores de extracto etéreo (EE), fibra bruta (FB), proteína bruta (PB) y extracto libre de nitrógeno (ELN, carbohidratos) del alimento a través de la siguiente ecuación (Batres Gracias, 2004):

$$
\begin{gathered}
E B=[9(E E)+4(F B)+4(P B)+4(E L N)] \times 10 \\
\text { Donde: } \\
\mathrm{EE}=\text { Extracto etéreo }(\%) \\
\mathrm{FB}=\text { Fibra bruta }(\%) \\
\mathrm{PB}=\text { Proteína bruta }(\%) \\
\mathrm{EB}=\text { Energía bruta }(\mathrm{cal} / \mathrm{g}) \\
\mathrm{ELN}=\text { Extracto libre de nitrógeno }(\%)
\end{gathered}
$$

\section{Estimación por método calorimétrico}

Para el método calorimétrico se usó la bomba calorimétrica IKA C 2000, que consiste en una cámara cerrada inyectada con oxígeno, en cuyo interior se combustiona la muestra de alimento mediante ignición con un conductor eléctrico. La cámara está sumergida en agua a temperatura ambiente y, al combustionar la muestra, provoca un aumento de temperatura del agua circundante. El valor de energía bruta es determinado directamente por el equipo (IKA, s.a.).

\section{Análisis Estadístico}

Los resultados de energía bruta de cada subproducto aplicando cada método, fueron analizados, para determinar sus estadígrafos descriptivos (medias, desviaciones estándar, varianzas), adicionalmente se aplicó el Test Anderson-Darling (Mohd Razali \& Bee Wah, 2011) para determinar si los valores obtenidos responden a una distribución Normal. 
Se definió como hipótesis nula que las medias de los resultados obtenidos por ambos métodos son similares, con un nivel de confianza del 95\%.

$$
\begin{aligned}
& \mathrm{H}_{\mathrm{o}}: \mu \mathrm{cal}=\mu \text { mat } \\
& \mathrm{H}_{\mathrm{a}}: \mu \mathrm{cal} \neq \mu \text { mat }
\end{aligned}
$$

Las características de cada conjunto de resultados: datos continuos o discretos, tamaño de muestras, tipo de distribución de resultados, homogeneidad de varianzas, y tipo de análisis (comparación de medias) se utilizaron para definir el tipo de test estadístico a aplicar (Minitab®, 2017).

\section{Resultados}

Los resultados de análisis químico proximal se indican en la Tabla 1:

Tabla 1. Resultados de determinación de energía bruta, por estimación matemática y por calorimetría.

\begin{tabular}{ccccc}
\hline Etiquetas de fila & $\begin{array}{c}\text { \% Extracto } \\
\text { etéreo }\end{array}$ & $\begin{array}{c}\text { \% Fibra } \\
\text { bruta }\end{array}$ & $\begin{array}{c}\text { \% Proteína } \\
\text { bruta }\end{array}$ & $\begin{array}{c}\text { \% Extracto } \\
\text { libre de } \\
\text { nitrógeno }\end{array}$ \\
\hline Tronco brócoli & 3,078 & 32,218 & 14,001 & 32,997 \\
Hoja zanahoria & 2,781 & 21,420 & 18,857 & 39,125 \\
Chaqui sara & 5,353 & 18,345 & 11,450 & 63,105 \\
Hoja de chocho & 3,423 & 18,455 & 26,225 & 38,768 \\
Hoja de maíz & 2,357 & 32,223 & 16,843 & 36,723 \\
Polvillo quinua & 3,860 & 19,678 & 13,943 & 52,038 \\
Hoja de camote & 1,938 & 20,845 & 16,920 & 43,865 \\
Hoja de plátano & 5,520 & 34,020 & 16,163 & 33,299 \\
\hline
\end{tabular}

En la Tabla 2, se indican los valores de energía determinados por ambos métodos, sus desviaciones estándar y sus varianzas.

Tabla 2. Resultados de energía, por estimación matemática y calorimetría

\begin{tabular}{ccc}
\hline Sub Producto & $\begin{array}{c}\text { Met. Calorimétrico } \\
\text { cal /g }\end{array}$ & $\begin{array}{c}\text { Met. Matemática } \\
\text { cal/g }\end{array}$ \\
\hline Tronco de brócoli & 3522,7 & 3445,6 \\
Hoja de zanahoria & 3629,1 & 3426,2 \\
Chaqui sara & 4288,5 & 4197,6 \\
Hoja de chocho & 4064,9 & 3646,2 \\
Hoja de maíz & 3868,2 & 3643,6 \\
Polvillo de quinua & 3779,2 & 3773,8 \\
Hoja de camote & 3825,0 & 3439,5 \\
Hoja de plátano & 4016,4 & 3836,3 \\
\hline
\end{tabular}

Los valores obtenidos presentaron las siguientes características para cada subproducto, y en ambos métodos:

- Datos continuos

- Muestra de tamaño moderado

- Distribución de frecuencias de tipo normal 
- Varianzas heterogéneas

Este conjunto de características llevó a elegir al Test t a dos colas, con variación de Welch (Welch, 1947), como método inferencial.

Los resultados de energía bruta se ingresaron y analizaron utilizando la aplicación informática Minitab 17 demo, aplicando el Test de Welch a dos colas y con un nivel de significancia del 5\% $(\alpha=0,05)$, de modo que la probabilidad P debería superar al valor $\alpha$ para considerar que se cumple la hipótesis nula. Se obtuvieron los siguientes resultados:

Tabla 3. Resultados test t de Welch, para hipótesis de similaridad de medias entre método calorimétrico y de estimación matemática

\begin{tabular}{cccccc}
\hline SUBPRODUCTO & $\mathbf{P}$ & $\boldsymbol{\alpha}$ & RELACIÓN & RESULTADO & OBSERVACIÓN \\
\hline Tronco de brócoli & 0,025 & 0,25 & $\mathrm{P}<\alpha$ & $\mathrm{Ho}_{1}$ se rechaza & $\mu \mathrm{cal} \neq \mu \mathrm{mat}$ \\
Hoja de zanahoria & 0,000 & 0,25 & $\mathrm{P}<\alpha$ & $\mathrm{Ho}_{1}$ se rechaza & $\mu \mathrm{cal} \neq \mu \mathrm{mat}$ \\
Chaqui Sara & 0,615 & 0,25 & $\mathrm{P}>\alpha$ & $\mathrm{Ho}_{1}$ se acepta & $\mu$ mat similar $\mu$ \\
& & & & & cal \\
Hoja de chocho & 0,000 & 0,25 & $\mathrm{P}<\alpha$ & $\mathrm{Ho}_{1}$ se rechaza & $\mu \mathrm{cal} \neq \mu$ mat \\
Hoja de maíz & 0,000 & 0,25 & $\mathrm{P}<\alpha$ & $\mathrm{Ho}_{1}$ se rechaza & $\mu \mathrm{cal} \neq \mu$ mat \\
Hoja de camote & 0,000 & 0,25 & $\mathrm{P}<\alpha$ & $\mathrm{Ho}_{1}$ se rechaza & $\mu \mathrm{cal} \neq \mu$ mat \\
Polvillo de quinua & 0,858 & 0,25 & $\mathrm{P}>\alpha$ & $\mathrm{Ho}_{1}$ se acepta & $\mu$ mat similar $\mu$ \\
Hoja de plátano & 0,015 & 0,25 & $\mathrm{P}<\alpha$ & $\mathrm{Ho}_{1}$ se rechaza & $\mu \mathrm{cal} \neq \mu \mathrm{mat}$ \\
\hline
\end{tabular}

Durante las mediciones se observó que los valores de energía bruta obtenidos por estimación matemática son menores respecto a los obtenidos por método calorimétrico. Para verificar esta observación se hizo un análisis a la totalidad de datos, comprobándose que la media de energía bruta por estimación matemática fue significativamente menor que la media de energía bruta por el método calorimétrico.

Figura 1. Prueba de la media de la totalidad de mediciones.

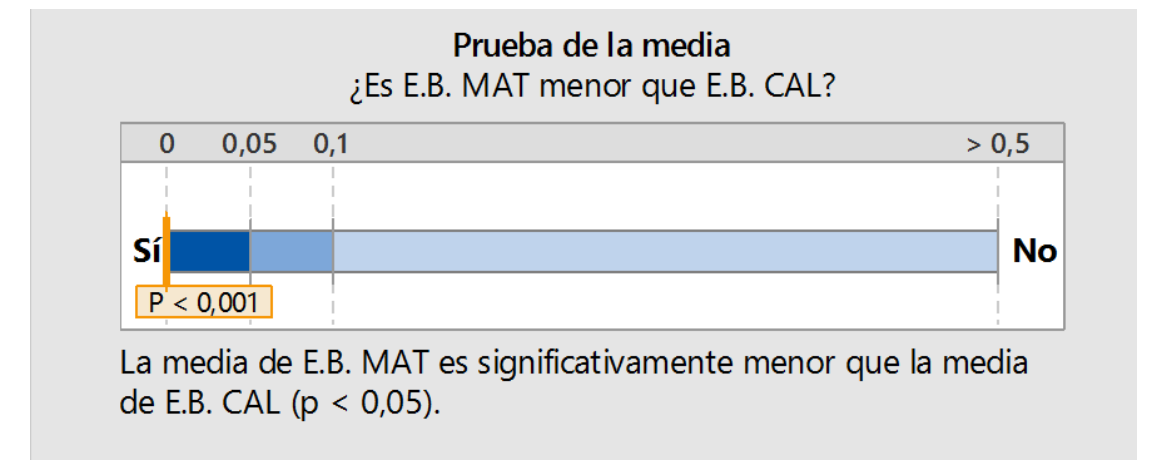

Fuente: Autores, Ecuador, 2017 


\section{Discusión}

En el presente estudio los valores de energía bruta obtenidos oscilan entre los 3175 y $4226 \mathrm{cal} / \mathrm{g}$ por el método de estimación matemática, y por el calorimétrico valores entre 3324 y $4340 \mathrm{cal} / \mathrm{g}$. Teniendo en consideración lo indicado por (Fillat, García-González, Gómez, \& Reiné, 2008), quienes establecen que, en promedio los vegetales poseen un valor de $4400 \mathrm{cal} / \mathrm{g}$ de energía bruta, y que el contenido energético de las plantas varía con el tiempo, aumentando en los tejidos maduros, puede afirmarse que los valores encontrados están dentro de lo estimado en la bibliografía. Por otro lado, la mediana de los valores de energía de estos subproductos, procedentes de la sierra ecuatoriana, 3644.9 cal, es menor a la mediana de la energía medida con subproductos propios de la costa ecuatoriana, 4619 cal (Torres Navarrete, y otros, 2017).

Respecto a la comparación de los valores de las medias, únicamente en los casos de los subproductos chaqui sara y polvillo de quinua se verificó igualdad estadística; mientras que en los otros seis sub productos el test muestra que los valores medidos por calorimetría no son estadísticamente iguales a los de la estimación matemática. En todos los casos, además se observó y confirmó la presencia de un sesgo positivo, donde los valores obtenidos por calorímetro son entre $4 \%$ y $10 \%$ mayores que los de estimación matemática.

Si bien no corresponde a este estudio el determinar la naturaleza y causas de este sesgo, algunas posibilidades incluyen: efecto del alto contenido de carbohidratos en alimentos de tipo harina, siendo éstos donde se verificaron medias estadísticamente iguales; contenido de extracto etéreo puesto que los componentes grasos se caracterizan por generar altas cantidades de energía al ser combustionados (MdDonald, Edwards, \& Morgan, 2006). Otra posibilidad es que la estimación calorimétrica, al medir calor generado por combustión, tome en cuenta energía producida por componentes no considerados en el cálculo que se aplica en la estimación matemática, tal sea el caso de la fibra, misma que, según (Cardona, y otros, 2002) hace que las materias primas y subproductos sean menos energéticos y menos digeribles. Un aspecto interesante, es que los dos subproductos que presentaron similaridad, también fueron aquellos con menor contenido proteico, y al mismo tiempo mayor cantidad de extracto libre de nitrógeno.

\section{Conclusión}

Los valores de energía bruta de subproductos agrícolas de la sierra ecuatoriana, obtenidos por estimación matemática presentan un sesgo respecto a los valores obtenidos por el método calorimétrico; siendo los últimos entre $4 \%$ y $10 \%$ mayores; con excepción de chaqui sara y polvillo de quinua, en los que puede considerarse que los resultados obtenidos por ambos métodos son estadísticamente similares. 
Adicional a la diferencia de medias, la existencia de un sesgo indica la necesidad de estudios posteriores para correlacionar el efecto de diferentes componentes de los subproductos en los valores de energía bruta.

\section{Referencias}

- Barrera, V., Tapia, C., \& Monteros, Á. (2004). Raíces y tubérculos andinos: Alternativas para la conservación y uso sostenible en el Ecuador. Quito, Ecuador: INIAP, CIP.

- Batres Gracias, R. A. (2004). Comparación entre el método de estimación matemática y el método de calorimetría para determinar el contenido energético del subproducto de galleta y de panadería para la alimentación de cerdos en desarrollo. Guatemala: Universidad San Carlos de Guatemala.

- Cañas Cruchaga, R., Aguilar González, C., García Gómez, F., Quiroz, R. A., \& otros. (1992). Simulación de sistemas pecuarios. San José: RISPAL.

- Caravaca Rodríguez, F. P., Castel Genís, J. M., \& Otros. (2005). Bases de la producción animal. Sevilla: Universidad de Sevilla.

- Cardona, M., Sorza, J., Posada, S., Carmona, J., Ayala, S., \& Álvarez, O. (2002). Establecimiento de una base de datos para la elaboración de tablas de contenido nutricional de alimentos para animales. Revista Colombiana de Ciencia Pecuarias, 15(2), 240-246.

- Fillat, F., García-González, R., Gómez, D., \& Reiné, R. (2008). Pastos del Pirineo. Madrid.

- García, M., Henry, T., \& Schulmeister, T. (2015). Nutrición animal en sistemas tropicales: Uso de residuos agrícolas en la producción animal. Maskana, ler Congreso Internacional de Producción Animal Especializada.

- Hernández, M., \& Sastre, A. (1999). Tratado de nutrición. Madrid: Días de Santos S.A.

- IKA. (s.f.). IKA Calorimeter System C2000 basic. IKA.

- MdDonald, P., Edwards, R., \& Morgan, C. (2006). Nutrición animal. Zaragoza, España: Editorial Acribia S.A.

- Mohd Razali, N., \& Bee Wah, Y. (2011). Power comparisons of Shapiro-Wilk, KolmogorovSmirnov, Lilliefors and Anderson-Darling Tests. Journal of Statistical Modeling and Analytics, 2(1), 21-33.

- Mora Brautigan, I. (2007). Nutrición animal (1ra ed.). San José, Costa Rica: EUNED.

- Núñez Torres, O. (2017). Utilización de alimentos no tradicionales y subproductos agrícolas en la nutrición animal. Journal of the Selva Andina Animal Science, 4(1), 1-2. 
- Posada, S., Rosero, R., Rodríguez, N., \& Costa, A. (2012). Comparación de métodos para la determinación del valor energético de alimentos para rumiantes. Revista MVZ Córdoba, 17(3), 3184-3192.

- Santos, R. (2010). O valor energético dos alimentos. Exemplo de uma determinação experimental, usando calorimetria de combustão. Quimica nova, 220-224.

- Torres Navarrete, E., Sánchez Laíño, A., Díaz Ocampo, R., Solórzano Robinson, M., Barrera Álvarez, A., \& Jácome López, G. (2017). Composición química de productos y subproductos agrícolas utilizados en alimentación animal por pequeños productores en la zona de Quevedo, Ecuador. Revista Amazónica Ciencia y Tecnología.

- Welch, D. (1947). The generalization of "Studnet's" problem when several different population varlances are involved. Biometrika, 34(1-2), 28-35. 


\section{PARA CITAR EL ARTÍCULO INDEXADO.}

Chugñay Cargua, A., Ramos Flores, M., Chugñay Cargua, M., \& Ramos Flores, M. (2019). Estudio comparativo entre el método calorimétrico y el de estimación matemática para la determinación energía bruta en subproductos agrícolas. Ciencia Digital, 3(3.4.), 19-28. https://doi.org/10.33262/cienciadigital.v3i3.4.831

\section{Liencia}

El artículo que se publica es de exclusiva responsabilidad de los autores y no necesariamente reflejan el pensamiento de la Revista Ciencia Digital.

El artículo queda en propiedad de la revista y, por tanto, su publicación parcial y/o total en otro medio tiene que ser autorizado por el director de la Revista Ciencia Digital.
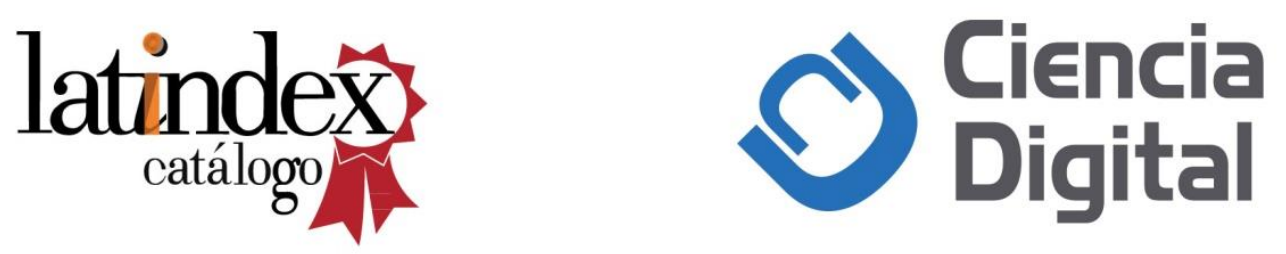